\title{
Is population based screening mammography starting at 40 justifiable? Benefits, risks and common sense
}

\section{Deepa Singal and Tannis Erickson}

\author{
University of Manitoba
}

In 2009, over 20,000 Canadian women were diagnosed with breast cancer and over 5,000 women died from breast cancer, demonstrating a profound burden to the health of the population ${ }^{1}$. Over the past two decades, screening mammography, which uses x-rays to find tumors in asymptotic women, has replaced clinical presentation as the principle means of detecting breast cancer and is currently being used throughout Canada ${ }^{2}$. In 2008, 74\% of women aged 50-69 received a screening mammogram ${ }^{3}$.

One of the most polarized debates among health professionals in recent years has been the value of screening mammography for women aged 40 to 49 . Past research examining the value of screening mammography, usually with women aged 50 and older, has shown positive results, including a decrease in breast cancer mortality by approximately $22 \%$. However, when reviewing the results for women aged 40 to 49 , we see less of a decrease in breast cancer deaths ${ }^{4}$. Moreover, experts believe that about half of this decrease is due to improved treatment strategies rather than early diagnosis screening mammography ${ }^{5}$.

The main reason for this debate is the difference we see in the effectiveness of mammography for women less than 50 years of age. Women under 40 have denser breast tissue, which decreases the sensitivity of mammography for detecting tumors. This test sensitivity is also decreased by the lower incidence of breast cancer in this age group $5,7,8$. In women aged $40-49,26 \%$ of cancers are not seen on mammograms, versus only $10 \%$ of cancers not seen in older women ${ }^{7}$.

The risks of screening mammography are also greater for women aged 40 to $49^{8}$. These risks include: increased radiation exposure, increased number of false positive mammograms and risk of overdiagnosis and treatment. A recent review of screening mammography reports a $30 \%$ rate of overdiagnosis and subsequent treatment of breast cancers ${ }^{6}$. Overdiagnosis occurs when screening picks up cancers that do not cause mortality or symptoms. Harm from overdiagnosis is particularly an issue for a certain kind of cancer, ductal carcinoma in situ (DCIS). Most cases of DCIS will not be associated with future invasive breast cancer but almost all women diagnosed will undergo lumpectomy and radiation therapy, some will even have a mastectomy ${ }^{5}$. These risks come with great psychological stress to these women and their families and are largely due to the greater number of mammograms they will have during their lifetime.

Less than $2 \%$ of women in their forties will develop breast cancer and most of these cases will be symptomatic, allowing for alternate means of diagnosis ${ }^{4}$. However, with routine screening mammography, all of these women would be exposed to the risks of increased screening. In a summary provided in Table 1, research shows that 40 year old women have more positive test results but fewer invasive breast cancers resulting in more false positive test results. They also have a significantly less gain in life expectancy than women in older age groups and, thus, decreased averted mortality.

Table 1: Estimated benefits \& risks of annual screening mammography for 10 years in 1,000 average women ${ }^{(5)}$

\begin{tabular}{|l|c|c|}
\hline & Aged 40 years & Aged 60 years \\
\hline Mammograms & 10,000 & 10,000 \\
\hline Positive test result & 550 & 390 \\
\hline Invasive breast cancer & 14 & 35 \\
\hline Breast cancer deaths averted & 0.3 & 1.4 \\
\hline Gain in life expectancy & 3 days & 20 days \\
\hline
\end{tabular}

This debate has been framed by some experts as evidence versus emotion; perhaps one life saved in women aged 40-49 is worth the risks that come with screening to the rest of the population. However, evidence demonstrates that as a population based intervention, screening mammography among women 40 to 49 years of age will not increase the life expectancy of the population or significantly decrease mortality. There are various ways to interpret the body of literature on breast cancer screening and this discord is evident within the medical community. Among the various organizations with published guideline statements regarding 
routine screening mammography, ${ }^{9,10,11,12,15,16,17,18,19,20}$ only the American College of Obstetricians \& Gynecologists and the American Cancer Society recommend routine screening mammography for women under the age of 50 .

Women with a family history or risk factors for breast cancer should discuss when to begin screening with their physician, however for asymptomatic women with no family history of breast cancer a population based screening program may cause more harm than benefit. Furthermore, in Canada where screening is covered by universal publicly-funded Medicare, screening younger women who are not at risk may take up scarce resources with little benefit, however; a costeffectiveness analysis is needed in this area to support resource allocation to this age group. Beyond popular public opinion and potential biases of health care professionals, assessment of screening effectiveness requires an objective evaluation of evidence that the benefits outweigh the risks in asymptomatic patients. Currently, there is no conclusive evidence that suggests that the benefits of screening mammography for asymptomatic women outweigh the risks. Therefore, implementing population health programs that include this age group are not likely to improve the overall health of the population and routine screening of women from age 40-49 should not be recommended ${ }^{5,12}$.

\section{References}

1. Canadian Cancer Society. Canadian Cancer Statistics 2009.

2. Schopper D, de Wolf C. How effective are breast cancer screening programmes by mammography? Review of the current evidence. European Journal of Cancer. 2009;45:1916-1923.

3. Statistics Canada. Mammography Statistics Table 105-0543, accessed from CANSIM March 29, 2010.

4. Armstrong K, Moye E, Williams S, Berlin JA, Reynolds EE. Screening Mammography for Women 40 to 49 Years of Age: A Systematic Review for the American College of Physicians. Ann Intern Med. 2007;146:516-526.

5. Tice JA, Kerlikowske K. Screening and Prevention of Breast Cancer in Primary Care. Prim Care Clin Office Pract. 2009;36:533-558.

6. Gotzsche PC, Nielson M. Screening for breast cancer with mammography (Review) The Cochrane Collaboration 2009, Issue 4.

7. Manitoba Brest screening program

8. Bjurnstam N, Bjorneld L, Warwick J, et al. The Gothenburg Breast Screening Trial. Cancer. 2003;Vol 97, No. 10: 2387-2396.

9. Miller AB, To T, Baines CJ, Wall C. Canadian National Breast Screening Study: 1. Breast cancer mortality after 11 to 16 years of follow-up. Annals of Internal Medicine. 2002;137(5 part 1):E305E315.

10. Qaseem A, Snow V, Sherif K, Aronson M, Weiss KB, Owens DK. Screening Mammography for Women 40 to 49 Years of Age: A Clinical Practice Guideline from the American College of Physicians. Ann Intern Med. 2007; 146:511-515.

11. International Journal of Gynecology \& Obstetrics .ACOG Practice Bulletin. Breast Cancer Screening. 2003;80:313-324.

12. Nelson HD, Tyne K, Naik A, Bougatsos C, Chan BK, Humphrey L. Screening for Breast Cancer: An Update for the US Preventive Services Task Force. Ann Intern Med. 2009;151(10):727-737.

13. Ringash J. Preventative health care, 2001 update: screening mammography among women aged 40-49 years at average risk of breast cancer. CMAJ Feb 20, 2001; 164(4):469-476.

14. Baines CJ. Are There Downsides to Mammography Screening? The Breast Journal.2005; Vol 11, Suppl 1:S7-S10.
15. Moss S. Should women under 50 be screened for breast cancer? British Journal of Cancer. 2004; 91:413-417.

16. Canadian Cancer Society Guidelines, www.cancer.ca/Canadawide/Prevention/Get $\% 20$ screened/Early $\% 2$ 0detection\%20and\%20screening\%20for\%20breast\%20cancer.aspx ? sc lang=en\&p=1, accessed March 17, 2010.

17. Canadian Medical Association, Guideline for the Early Detection of Breast Cancer, www.topalbertadoctors.org/informed practice/ clinical practice guidelines/complete\%20set/Breast\%20Cancer/bre ast cancer_guideline.pdf , accessed on March 17, 2010.

18. American Academy of Family Physicians, FPIN's Clinical Inquiries, www.aafp.org/afp/2004/1101/p1750.html, accessed on March 15, 2010

19. US Preventive Services Task Force. Screening for Breast Cancer: US Preventive Task Force Recommendation Statement. Annals of Internal Medicine. 2009:151(10): 716-726.

20. American Cancer Society. Guidelines for the Early Detection of Cancer. www.cancer.org/docroot/ped/content/ped 2 3x acs cancer detecti on_guidelines 36.asp, accessed March 17, 2010.

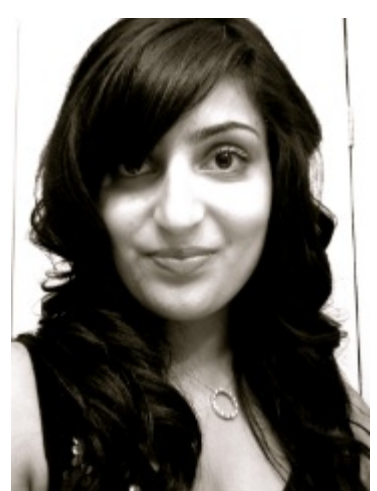

\section{Author Profiles}

Deepa Singal is a $\mathrm{PhD}$ Student at the University of Manitoba in the Department of Community Health Sciences. Her dissertation will investigate the characteristics and health care utilization of women who give birth to children with FASD. Her research interests included health services and policy research, specifically in primary health care delivery, health and mental health of at risk children including children in care and children with FASD. She is a member of the Western Regional Training Centre Graduate Program and is a recipient of a Manitoba Health Research Council funding award.

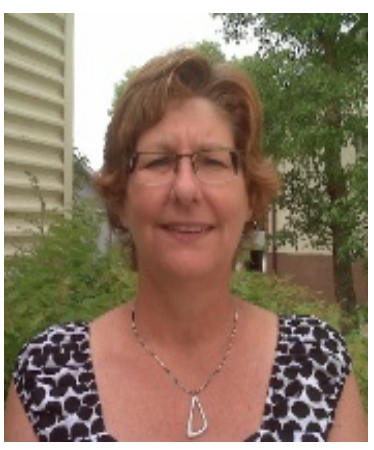

Tannis Erickson is an MSc candidate at the University of Manitoba (Department of Community Health Sciences) studying chronic disease risk factors in Manitoba youth. Her research interests include predictors of positive health behaviors in youth and effectiveness of health promotion programs. 